\title{
A Self-Efficacy-Enhancing Intervention for Chinese Patients after Total Hip Arthroplasty: Study Protocol for A Randomized Controlled Trial with 6-Month Follow-Up
}

\section{Bo Deng ( $\nabla$ dengbo@sysucc.org.cn )}

Sun Yat-Sen University Cancer Prevention and Treatment Center: Sun Yat-sen University Cancer Center

Yu mei Chen

Foshan Hospital of Traditional Chinese Medicine

Mei fen Zhang

Sun Yat-sen University School of Nursing https://orcid.org/0000-0001-7931-3285

\section{Ya Meng}

Sun Yat-sen University School of Nursing

\section{Yi heng Zhang}

Sun Yat-sen University School of Nursing

\section{Xing xian Tan}

Foshan Hosipital of Traditional Chinese Medicine, Department of Orthopedics

\section{Xiao hong Zhou}

Foshan Hosipital of Traditional Chinese Medicine, Department of Orthopedics

\section{Research Article}

Keywords: Total hip arthroplasty, Self-efficacy, Exercise adherence, Rehabilitation

Posted Date: May 26th, 2021

DOI: https://doi.org/10.21203/rs.3.rs-371615/v1

License: (c) (i) This work is licensed under a Creative Commons Attribution 4.0 International License. Read Full License

Version of Record: A version of this preprint was published at Journal of Orthopaedic Surgery and Research on January 4th, 2022. See the published version at https://doi.org/10.1186/s13018-021-026898. 


\section{Abstract}

Background: Total hip arthroplasty (THA) is a common and effective surgical method for advanced hip arthritis. Rehabilitation exercises are important to improve joint function after THA and are usually conducted in a home-based program. Poor patient adherence limits improvements in pain and function, affecting quality of life. The increasing use of THA in the aging Chinese population underscores the need to develop strategies that maximize functional outcomes. The purpose of this pilot study is to develop and assess the feasibility of a self-efficacy-enhancing intervention (SEEI) to improve exercise adherence in patients undergoing THA.

Methods: This single-blinded, parallel, randomized control trial will recruit 150 patients after THA and randomly assign them to an intervention or control group using computer-generated block randomization. The control group will receive usual care using evidence-based guidelines. The intervention group will receive the 6-month SEEI comprising personalized exercise guidance and self-efficacy education delivered using one face-to-face education session and four telephone consultations, supplemented by written materials. Participants are encouraged to build confidence in their own abilities, set rehabilitation goals, and self-monitor their physical exercise.

Results: Assessments will be conducted at baseline, and 1, 3, and 6 months postsurgery. The outcome indicators are exercise adherence, physical function, anxiety and depression, self-efficacy of rehabilitation, joint function, and quality of life.

Conclusions: This study will test a theory-based intervention program to improve self-efficacy in rehabilitation, which may significantly impact out-of-hospital rehabilitation. The results will provide evidence to inform the postoperative recovery of patients undergoing THA or similar procedures.

Trial registration: Chinese Clinical Trials Registry (ChiCTR2000029422), registered 31 January 2020.

\section{Background}

Total hip arthroplasty (THA) is a major surgical procedure performed worldwide [1]. Approximately 400,000 THAs are performed annually in China [2], and this is growing rapidly due to the high level of patient needs and the challenges of an aging population [3,4]. THA is a cost-effective intervention that reduces or even eliminates pain [5]. Despite these encouraging facts, differences remain in patient selfreported functional improvement after THA [6, 7]. Some studies have reported significant positive correlations between the surgical effects in patients after hip/knee arthroplasty and exercise adherence [8-11]. Home exercise adherence is usually high in the early stage but gradually decreases over time [12, 13]. As poor adherence to home exercise is common and affects therapeutic effectiveness and healthcare costs [14], there is an urgent need for economic, achievable, and effective interventions to improve home exercise adherence [15]. 
Self-efficacy is an important part of promoting healthy behavior and a significant determinant influencing the initiation and maintenance of positive health behaviors [16, 17]. The concept of self-efficacy was first proposed in 1977 [18]. According to self-efficacy theory, if individuals believe that they can achieve results, then they become more active and in control of their lives. Therefore, for rehabilitation after THA, patients must believe in their ability to perform specific physical activities (i.e., the patients must have self-efficacy) and must be aware of the motivation to do so. A high sense of individual self-efficacy can lead to positive health behaviors $[18,19]$. A higher sense of self-efficacy indicates that individuals will exhibit good exercise adherence, while a lower sense of self-efficacy is regarded as an obstacle to ensuring effective rehabilitation [20].

A number of studies have shown that enhancing self-efficacy is helpful to patient self-management and promotes health-related behaviors and good outcomes. Related interventions have been successfully applied in people with coronary heart disease [21], diabetes [22], hypertension [23], stroke [24], and arthritis [25], and the rehabilitation of patients undergoing joint replacement is significantly related to selfefficacy $[26,27]$. Previous investigations have shown moderate self-efficacy of Chinese patients after hip and knee arthroplasty $[28,29]$. Nevertheless, studies in this field are scarce, the majority used small sample sizes, and self-efficacy has not been considered in rehabilitation program development, leading to substantial extension of rehabilitation times and unsatisfactory outcomes.

The theory of self-efficacy provides a good basis for nursing interventions for patients after THA. This study is guided by the self-efficacy theory of Bandura, which posits that enhancement of self-efficacy is beneficial for patient rehabilitation and for achieving optimal outcomes. To investigate this further, we developed a 6-month self-efficacy-enhancing intervention (SEEI), to support adherence to prescribed home-based exercise for patients following THA. The SEEI was developed in rigorous accordance with Bandura's self-efficacy theory and is a complex intervention measure. In the present study, self-efficacy is in the context of rehabilitation following THA.

Our primary hypothesis is that adherence to the prescribed home exercise program in the 6 months after THA will be higher in the group receiving the SEEI compared with the group receiving usual care. Our second hypothesis is that the SEEI group will exhibit greater improvements in other outcomes (e.g., pain, function, health-related quality of life [QOL], anxiety and depression, and another measure of exercise adherence) than the control group.

\section{Methods}

\section{Study design}

This prospective randomized controlled trial is to test the effect of a nurse-led SEEl, based on the theory of self-efficacy, on exercise compliance of patients after THA. All participants are randomly assigned to the SEEl or usual care (control) group. This study will examine the effect of the intervention on patient exercise compliance after THA and evaluate the contribution of the intervention to the improvement of 
joint function. We hypothesize that participants in the experimental SEEI group will show significantly better exercise self-efficacy, a greater increase in exercise compliance, superior hip function, lower levels of anxiety and depression, and increased QOL. Data will be assessed at baseline and 1, 3, and 6 months after surgery. The study has been approved by the Ethics Committee of our institution and will be registered, constructed, and presented following the recommendations of the CONSORT statement [30] and SPIRIT guidelines [31] (see Fig. 1).

\section{Setting and population}

The study setting is the Joint Surgery Unit of the Orthopedics Department at a hospital.

Patients on the surgical waiting list for THA will be invited to participate in the study after preoperative assessment. Men and women aged 18 years or older and scheduled for primary, single-side THA surgery at the Joint Surgery Unit are eligible for participation. Trial inclusion criteria are as follows: participants must be able to read and understand Chinese, willing to participate in a 6-month exercise intervention, and provide informed consent. Participants are ineligible if they meet one or more of the following exclusion criteria: (i) an unstable medical condition due to fracture, malignancy, infection, or failure of THA; (ii) inability to provide informed consent due to mental disorder, dementia, or cognitive impairment that prevent completion of self-report surveys; (iii) co-existing conditions that would negate functional improvement with surgery and exercise (e.g., severe Parkinson disease, or hemiparesis); (iv) simultaneous bilateral THA; (v) surgery for neoplastic disease or imminently scheduled surgery and unavailable to complete the study procedures; or (vi) planning another THA or total knee replacement within 6 months.

\section{Development of the SEEl program}

\section{Preparation phase}

After reviewing international guidelines on the development of exercise interventions for adults undergoing hip surgery, the preparation of the SEEl program followed a three-stage process:

1. Setting up a nurse-led intervention team, mainly composed of orthopedic nursing clinical specialists, orthopedic surgeons, orthopedic physicians, senior orthopedic nurses, and research assistants, to control the overall research direction, formulate and evaluate intervention programs, and control the quality of research. A draft intervention was produced after a series of interviews with patients undergoing THA by experienced healthcare professionals; the specific content and protocol were subsequently validated by a panel of orthopedic experts.

2. Formulating the "Handbook for Rehabilitation after discharge following THA" including two main aspects: post-discharge guidance, including general information about home reorganization, functional exercise, matters or follow-up needing attention, and daily life guidance during each rehabilitation period after THA; and a functional exercise diary in the rehabilitation manual that is convenient for patients to record their exercises and any problems. The functional exercise and daily life guidance sections contain illustrations and detailed written explanations. Contact information for 
the department, orthopedic specialist, and orthopedic nurse is at the back of the guide. The handbook will be available for patients in both groups.

3. Registering a WeChat account for research and filming a functional exercise video. A WeChat account has been created specifically for the experimental group and is managed by a research assistant, as a convenient means to establish multiple contacts with patients and reduce follow-up loss. WeChat is used to promote exercise videos provided for patients and their families to learn about the various stages of joint replacement rehabilitation. WeChat will also be used to collect video data, including of exercise and walking, during the rehabilitation process.

\section{Intervention theoretical framework}

The intervention is based on Bandura's self-efficacy theory [32], combined with social cognitive theory (SCT) [33]. Consistent with SCT, interventions target self-management knowledge, attitudes (selfefficacy), and behavioral abilities through a variety of printed materials and activities. The intervention program is also conducted according to Bandura's self-efficacy theory [34], which includes four main aspects: individual past experience, witnessing the experiences of other individuals, verbal motivation from others, and physical and emotional support for patients, specific to the particular behavior [35]. The four strategies recommended by Bandura [36] have been incorporated into each component of the intervention. Individual past experience includes emphasizing the importance of functional exercise on the basis of the existing THA functional exercise table, helping participants to set achievable goals and actions, and encouraging participants to observe and record their exercise behavior and constantly accumulate self-management experience, according to their subjective physical experience. Vicarious experience includes sharing successful examples of patients who have experienced similar symptoms and recovered using self-management skills, to help participants build confidence, and encourage them to adhere to the functional exercise program. Verbal persuasion includes describing the benefits of exercise adherence to joint function, encouraging and acknowledging the participant's ability to deal with symptoms, and helping those who encounter difficulties to find the causes and propose corresponding solutions. Psychological monitoring includes the explanation of possible symptoms during postoperative rehabilitation and the discussion of treatment strategies. Patients are supported in these four strategies during training sessions. Table 1 outlines examples of the four strategies [18]. 
Table 1

SEEl components, strategies, and techniques

\begin{tabular}{|c|c|c|}
\hline Components & Strategies & Specific techniques \\
\hline \multirow[t]{4}{*}{$\begin{array}{l}\text { Individual past } \\
\text { experience }\end{array}$} & $\begin{array}{l}\text { - Setting achievable } \\
\text { goals }\end{array}$ & $\begin{array}{l}\text { - Consulting with patients to develop functional exercise } \\
\text { goals at different stages; making plans on when, where, } \\
\text { and how to conduct regular physical activities }\end{array}$ \\
\hline & $\begin{array}{l}\text { - Providing } \\
\text { information on the } \\
\text { benefits of }\end{array}$ & $\begin{array}{l}\text { - Providing information on the risk factors of a sedentary } \\
\text { lifestyle and the advantages of functional exercise }\end{array}$ \\
\hline & \multirow{2}{*}{$\begin{array}{l}\text { - Providing positive } \\
\text { feedback }\end{array}$} & $\begin{array}{l}\text { - Identifying postoperative rehabilitation challenges } \\
\text { through discussion }\end{array}$ \\
\hline & & - Providing positive feedback on patient accomplishments \\
\hline \multirow{2}{*}{$\begin{array}{l}\text { Vicarious } \\
\text { experience }\end{array}$} & \multirow{2}{*}{$\begin{array}{l}\text { Sharing cases of } \\
\text { successful } \\
\text { rehabilitation }\end{array}$} & - Sharing previous success stories to build confidence \\
\hline & & $\begin{array}{l}\text { - Introducing the successful experiences of others to } \\
\text { encourage patients to complete physical activities in the } \\
\text { following months }\end{array}$ \\
\hline \multirow{4}{*}{$\begin{array}{l}\text { Verbal } \\
\text { persuasion }\end{array}$} & \multirow{4}{*}{$\begin{array}{l}\text { Verbal } \\
\text { encouragement, } \\
\text { explanation, and } \\
\text { persuasion }\end{array}$} & - Describing the benefits of physical activities \\
\hline & & - Asserting that patients have the ability to self-manage \\
\hline & & $\begin{array}{l}\text { - Providing positive feedback on the patient's efforts and } \\
\text { giving verbal encouragement }\end{array}$ \\
\hline & & $\begin{array}{l}\text { - Reinforcing the past and present successes or } \\
\text { accomplishments of patients }\end{array}$ \\
\hline \multirow{3}{*}{$\begin{array}{l}\text { Supporting } \\
\text { patients } \\
\text { physically and } \\
\text { emotionally }\end{array}$} & \multirow{2}{*}{$\begin{array}{l}\text { - Developing } \\
\text { strategies to cope } \\
\text { with barriers }\end{array}$} & - Assessing patient expression of anxiety and depression \\
\hline & & $\begin{array}{l}\text { - Identifying individual barriers to and resources for } \\
\text { physical activity }\end{array}$ \\
\hline & $\begin{array}{l}\cdot \text { Help to seek social } \\
\text { support }\end{array}$ & $\begin{array}{l}\text { - Providing strategies for dealing with barriers and coping } \\
\text { in the future (postsurgery; significance of social support) }\end{array}$ \\
\hline
\end{tabular}

\section{Intervention structure and content}

Challenges to successful post-THA functional achievement were identified, and strategies to address them were developed and integrated to design the intervention. Telephone interview was selected as the primary basis for the intervention because it is a low-cost, wide-coverage mechanism that can provide support intervention after THA for patients with limited mobility and transport difficulties [37]. Although this format has not been thoroughly investigated in patients with THA, it has been well-studied for patients with other conditions and used alone or in combination with face-to-face educational sessions and print materials $[38,39]$. The initial intervention was further refined following a pilot pretest involving five patients.

The final intervention protocol includes a total of five sessions: one hour of face-to-face education before discharge, and four additional sessions post-discharge between 1 and 6 months after surgery. Except for 
the hospital visit, all visits are telephone based. The first call is approximately $40 \mathrm{~min}$, with follow-up contacts ranging from 20 to $30 \mathrm{~min}$. The intervention aims to enhance exercise adherence through the implementation of self-efficacy-enhancing strategies.

The initial face-to-face intervention is conducted in the hospital office before patients are discharged from the hospital after THA surgery. The focus of the initial session is to assess the functional exercise status and psychological condition of each individual, including the level of pain, the occurrence of complications, and knowledge of functional exercise. The nurse provides participants with the Rehabilitation Handbook after THA and instructs them to read it. The manual is used to supplement the face-to-face educational session. Participants are encouraged to refer to the manual at home for information. The nurses also provide video recordings of functional exercise after THA and teach patients the functional exercise movements and matters needing attention in daily life after surgery, as well as persuading and encouraging participants to set rehabilitation goals to be achieved one month post-THA, and mobilizing the encouragement and support of the participant's family members.

During the 6-month follow-up period, four subsequent health-coaching sessions are conducted via telephone or the WeChat app. These sessions are designed to enhance participant compliance with functional exercises and are guided by a protocol. Participants in each follow-up are required to selfreport the number of home exercises they have completed in the previous week, their level of pain, and their mental state. Nurses encourage and provide reinforcement of the participant's efforts and success, and empower them through support. Participants with a low adherence ( $<3$ exercise sessions/week) are then asked to select a barrier from a scheduled list (forgot, too tired, injured so unable to exercise, fear of movement, exercise inconvenience, lack of time, life stress) to explain why they were unable to complete the exercise as required. The nurse then provides a suggestion tailored to help overcome that barrier. According to the needs of each patient, the duration of each phone call ranges from 20 to $30 \mathrm{~min}$.

Four senior orthopedic nurses with bachelor degrees or above and at least 10 years of orthopedic nursing experience have been selected in the target hospital. All nurses have a specialist diploma, are very familiar with the physical and psychological problems and rehabilitation needs of patients after THA, and establish close relationships with the patients. Simultaneously, two research assistants are assigned to conduct the interventions with the patients, primarily through collection of questionnaire data, and assist with relevant intervention content. The two research assistants are very familiar with the care of patients undergoing joint replacement. Two days of training are provided for the nurses before intervention implementation, primarily including: communication skills, consistency of the intervention program, strategies to encourage physiological and psychological changes, detailed descriptions of problems that occur in the rehabilitation process after THA, dos and don'ts for the telephone follow-up, and matters included in the intervention follow-up record form. All staff also receive training on the study protocol and the procedures for collecting informed consent and data assessment. The principal investigator will monitor the conduct of the intervention through observation sampling sessions.

\section{Assessment of outcomes}


All assessment sessions (baseline [time 1], 1 month [time 2], 3 months [time 3], and 6 months [time 4] post-surgery) include measurement of the primary and secondary outcomes. A series of questionnaires related to health outcomes of the SEEl is implemented. During the baseline assessment, additional demographic information including health, medical history, and general health status is collected.

\section{Primary outcomes}

Self-efficacy for rehabilitation (SER) outcome scale

The 12-item SER was developed by Waldrop et al. [40], following Bandura's guidelines [33] to assess participant beliefs about their ability to perform behaviors typical in physical rehabilitation for knee and hip surgery. The SER was developed in conjunction with rehabilitation psychologists and physical and occupational therapists. Items increase in difficulty (e.g., items assessing self-belief in ability to stretch a leg, to those assessing beliefs in one's ability to walk). Items measure belief in ability to perform behaviors in varying therapy situations, including when experiencing pain and emotional distress. For each item, participants use an 11-point Likert scale, ranging from 0 (I cannot do) to 10 (certain I can do) to describe their confidence. The full score for the items is 120 , and higher scores indicate more perceived self-efficacy for rehabilitation. According to Bandura [33], "efficacy scores are summed and then divided by the total number of items to indicate the strength of perceived self-efficacy for the activity domain." Thus, mean self-efficacy scores were calculated. In this study, the reliability and validity of the Chinese version SER outcome scale will be assessed by six orthopedic nurses. The Cronbach's alpha reliability coefficient value for the total scale was 0.942 .

\section{Functional exercise compliance scale for THA patients}

The self-designed exercise compliance questionnaire was developed by Xinxin Li of Sun Yat sen University [29]. It can be divided into three dimensions: physical exercise compliance, exercise monitoring compliance, and initiative-seeking advice compliance. Each item is rated on a 4-point scale (4 points = "always do," 3 points = "almost always do," 2 points = "occasionally do," and 1 point = "never do"). The sum of item scores is the total score, with higher total scores indicating a greater level of compliance level with functional exercise. In this study, Cronbach's alpha ranges from 0.915 to 0.947 , with an intrarater reliability value of 0.92 .

\section{Secondary outcome measures}

Overall hip pain

Average overall hip pain in the past week is self-assessed using a 11-point numeric rating scale with score 0 representing "no pain" and 10 representing "extreme pain" (e.g., "pain as bad as you can imagine" or "worst pain imaginable") [41-43]. Participants select the whole number (0-10) that best reflects the intensity of their pain.

Activity and participation (WHO-DAS II) 
The World Health Organization Disability Assessment Schedule II (WHO-DAS II) was developed based on the concepts and theoretical models of the 2001 International Classification of Functioning, Disability and Health, regarding the general evaluation and measurement of health conditions, disabilities, and psychometric variables [44]. The WHO-DAS II provides a standardized cross-cultural method for measuring the health and disability status of adults (aged over 18 years) over a 30-day period and contains 32 items covering six domains: cognition, mobility, self-care, getting along with people, activities of daily life, and social participation [45]. Scores are classified into five levels: no difficulty, mild difficulty, moderate difficulty, severe difficulty, and extreme difficulty [46]. The total score range is $0-100$, with higher scores indicating higher limitations in daily life. In this study, the reliability of Cronbach's alpha and intraclass correlation coefficient in the WHO-DAS II are 0.73-0.99 and 0.8-089, respectively, illustrating that the Chinese version is suitable to assess activity and participation [47].

Anxiety and depression

Anxiety and depression are measured using the Chinese version of the Hospital Anxiety and Depression Scale (C-HADS) [48]. The HADS is a self-report questionnaire designed to assess depression and anxiety and has been widely used among patient groups [49]. This instrument consists of 14 items ( 7 for anxiety [HAD-A] and 7 for depression [HAD-D]), which are used as two separate measures of psychological disturbance. Each subscale is scored from 0 to 21, with higher scores indicating greater distress. The psychometric properties of C-HADS have been confirmed, with satisfactory results in terms of reliability and validity [48]. In this study, the Cronbach's alpha reliability coefficients for both the anxiety and depression subscales are both 0.85 [50].

Hip function

Hip function is monitored using the Harris Hip Score (HHS) for hip arthroplasty, a multidimensional disease-specific observational assessment of functioning, containing eight items representing pain, walking function, activities of daily living, and range of motion of the hip joint [51]. The HHS is widely used by physicians to assess physical function and pain relief in patients with hip disease [52]. The maximum score for the HHS is 100 points, with maximum possible scores for its components as follows: pain (44 points), function (47 points), range of motion (5 points), and deformity (4 points) [53]. A higher HHS score indicates better function.

QOL

Physical and mental health are assessed using the 12-item Short Form Health Survey (SF-12), a validated measure of QOL following joint replacement [54]. The SF-12 comprises 12 items for assessing physical and mental health and yields two summary scores: the physical and mental health composite scores [55]. Scoring is norm-based, with a mean of $50(S D=10)$; lower SF-12 scores indicate poorer physical and/or mental health. The Chinese versions of SF-12 components are established as reliable and valid in older patients [56].

\section{Sample size, statistical analysis, and monitoring}


The required sample size was determined as 64 patients in each group based on a previous study [57]. The study that our sample size calculations are based on experienced a low withdrawal rate (10\%). We have made a conservative estimate of a $15 \%$ withdrawal rate; therefore, we intend to recruit 150 participants.

\section{Randomization and blinding}

After completing all baseline data collection measures, participants are randomly divided into the intervention and control groups using computer-generated random numbers. Participants are assigned a series of continuous integers in the order of admission. To hide randomization, the independent researcher has prepared consecutively numbered, sealed, opaque envelopes, which are kept in a locked location. To avoid affecting exercise adherence behavior, participants are not informed of the two separate studies, re-randomization into this trial, or the exact details of the intervention. This process ensures adequate concealment, limiting the possibility of selection deviation. The statistician will be blinded to group allocation, which will be revealed upon completion of the statistical analyses.

\section{Data analysis}

IBM SPSS 25.0 will be used to analyze the collected data, according to the intent-to-treat principle. An alpha level of 0.05 will be applied for all statistical tests, and a $95 \%$ confidence interval used for all estimated values. Missing data will be managed using the last-observation-carried-forward method; this imputation method replaces missing values in the post-baseline follow-up with the last observed value for each individual. Descriptive statistics and frequency distributions will be generated for patient demographic and disease-related characteristics. Independent $t$ tests and $\chi^{2}$ analyses will be performed to evaluate whether there are significant differences in demographic or clinical characteristics and baseline measures between the control and intervention groups. A repeated measures multivariate analysis of variance will be used to test the effects of group assignments (intervention vs. control), time (pretest vs. post-tests) and group by time interaction, for the four outcome measures. If the assumption of sphericity is not met, the degrees of freedom for the $F$ ratio will be adjusted according to GreenhouseGessler epsilon. $P$-values $<0.05$ will be considered statistically significant for all comparisons.

\section{Monitoring}

The trial coordinator and lead investigators meet every 2 weeks to monitor adverse events and any issues relating to the trial, and to review recruitment and trial progress.

\section{Discussion}

This study protocol describes the theoretical basis and design of an ongoing randomized controlled trial that will test the effectiveness of a patient SEEI on THA functional outcomes. THA is a common and 
widely accepted treatment for advanced hip arthritis, and maximum improvement of joint function is a top priority. If effective, such interventions have great potential for dissemination. In addition to their potential to enhance patient rehabilitation self-efficacy, the lessons learned in this trial can provide guidance for tailored rehabilitation strategies after THA, including family activities and exercise programs.

\section{Limitations}

The authors acknowledge the following limitations: this is a single-center trial with no cost-effectiveness analysis and limited patient and public involvement in the design of the intervention.

\section{Declarations}

\section{Availability of data and materials}

Not applicable.

\section{Acknowledgements}

We would like to thank all the parents who participated into the study. We gratefully acknowledge Medical Scientific Research Foundation of Guangdong Province and Education Ministry of China for supporting this program..

\section{Authors' contributions}

Bo Deng, Yumei Chen, and Meifen Zhang conceived the idea and designed the experiments. Bo Deng and Yumei Chen led the experiments (with assistance from Ya Meng,Yiheng Zhang, Xiaohong Zhou and Xingxian Tan). Bo Deng, Ya Meng, Yiheng Zhang and Meifen Zhang contributed to data analysis and interpretation. Bo Deng, Ya Meng and Yiheng Zhang wrote the manuscript, Meifen Zhang revised the paper and all authors provided feedback.

\section{Funding}

This research was supported by Medical Scientific Research Foundation of Guangdong Province [grant 20160910], Education Ministry of the People's Republic of China [grant 18YJA840022]. The funders had no part in designing the study and in its implementation, analysis, data interpretation, presentation of the results and decision to submit a report for publication.

\section{Ethics declarations}

\section{Ethics approval and consent to participate}

The relevant sponsor has reviewed the study protocol and consent form, and it was prospectively approved by the Institution Review Board of the Sixth Affiliated Hospital of Sun Yat-sen University ( No: 
2020ZSLYEC-062).

\section{Consent for publication}

Not applicable.

\section{Competing interests}

The authors report no conflict of interest concerning the materials or methods used in this study stated in this manuscript.

\section{References}

1. Chechik $\mathrm{O}$, Khashan M, Lador R.. Surgical approach and prosthesis fixation in hip arthroplasty world wide. Arch Orthop Trauma Surg. 2013;133(11):1595-600.

2. Lei Z, Jianning Z. Prevention of complications after total hip arthroplasty. Chin J Orthop Traumatol. 2018;31(12):6-10.

3. Otten R, van Roermund PM, Picavet HS. [Trends in the number of knee and hip arthroplasties: considerably more knee and hip prostheses due to osteoarthritis in 2030]. Ned Tijdschr Geneeskd. 2010;154(154):A1534.

4. Kurtz S, Ong K, Lau E, Mowat F, Halpern M. Projections of primary and revision hip and knee arthroplasty in the United States from 2005 to 2030. J Bone Joint Surg Am. 2007;89(4):780-5.

5. Lavernia CJ, lacobelli DA, Brooks L, Villa JM. The Cost-Utility of Total Hip Arthroplasty: Earlier Intervention, Improved Economics. J Arthroplasty. 2015;30(6):945-9.

6. Rosal MC, Ayers D, Li W, Oatis C, Borg A, Zheng H, et al. A randomized clinical trial of a peri-operative behavioral intervention to improve physical activity adherence and functional outcomes following total knee replacement. BMC Musculoskelet Disord. 2011;12(1):226.

7. Ayers DC, Franklin PD, Trief PM, Ploutz-Snyder R. Freund D. Psychological attributes of preoperative total joint replacement patients: implications for optimal physical outcome. J Arthroplasty. 2004;19(7 Suppl 2):125-30.

8. Pisters MF, Veenhof C, Schellevis FG, Twisk JW, Dekker J, De Bakker DH.. Exercise adherence improving long-term patient outcome in patients with osteoarthritis of the hip and/or knee. Arthritis Care Res (Hoboken). 2010;62(8):1087-94.

9. Jensen C, Roos EM, Kjaersgaard-Andersen P, Overgaard S. The effect of education and supervised exercise vs. education alone on the time to total hip replacement in patients with severe hip osteoarthritis. A randomized clinical trial protocol. BMC Musculoskelet Disord. 2013;14:21.

10. Liu CJ, Latham NK. Progressive resistance strength training for improving physical function in older adults. Cochrane Database Syst Rev. 2009;6(3):244-6.

11. Latham NK, Bennett DA, Stretton CM, Anderson CS. Systematic review of progressive resistance strength training in older adults. J Gerontol A Biol Sci Med Sci. 2004;59(1):48-61. 
12. Campbell R, Evans M, Tucker M, Quilty B, Dieppe P, Donovan JL. Why don't patients do their exercises? Understanding non-compliance with physiotherapy in patients with osteoarthritis of the knee. J Epidemiol Community Health. 2001;55(2):132-8.

13. Lonsdale C, Hall AM, Murray A, Williams GC, McDonough SM, Ntoumanis N, et al. Communication Skills Training for Practitioners to Increase Patient Adherence to Home-Based Rehabilitation for Chronic Low Back Pain: Results of a Cluster Randomized Controlled Trial. Arch Phys Med Rehabil. 2017;98(9):1732-43.

14. Gordon R, Bloxham S. A Systematic Review of the Effects of Exercise and Physical Activity on NonSpecific Chronic Low Back Pain. Healthcare (Basel). 2016;4(2):22.

15. van Gool CH, Penninx BW, Kempen GI, Rejeski WJ, Miller GD, van Eijk JT, et al. Effects of exercise adherence on physical function among overweight older adults with knee osteoarthritis. Arthritis Rheum. 2005;53(1):24-32.

16. Fisher WA, Fisher JD, Harman J. The Information-Motivation-Behavioral Skills Model: A General Social Psychological Approach to Understanding and Promoting Health Behavior. Oxford: Blackwell Publishing Ltd; 2003.

17. Jeng C, Braun LT. The influence of self-efficacy on exercise intensity, compliance rate and cardiac rehabilitation outcomes among coronary artery disease patients. Prog Cardiovasc Nurs. $1997 ; 12(1): 13$.

18. Bandura A. Self-efficacy: toward a unifying theory of behavioral change. Adv Behav Res Ther. 1977;84(4):139-61.

19. Bandura A. Health Promotion by Social Cognitive Means. Health Educ Behav. 2004;31(2):143-64.

20. Tomasich A, Ferrario SR, Guzzi L, Lascioli R, Passante K.. Perceived self-efficacy and respiratory rehabilitation. G Ital Med Lav Ergon. 2006;28(1 Suppl 1):29-33.

21. Ruiz BA, Dibble SL, Gilliss CL, Gortner SR. Predictors of general activity 8 weeks after cardiac surgery. Appl Nurs Res. 1992;5(2):59-65.

22. Wu SF, Courtney M, Edwards H, McDowell J, Shortridge-Baggett LM, Chang PJ. Self-efficacy, outcome expectations and self-care behaviour in people with type 2 diabetes in Taiwan. J Clin Nurs. 2007;16(11C):250-7.

23. Warren-Findlow J, Seymour RB, Brunner HL. The association between self-efficacy and hypertension self-care activities among African American adults. J Community Health. 2012;37(1):15-24.

24. Robinson-Smith G, Johnston MV. Allen J. Self-care self-efficacy, quality of life, and depression after stroke. Arch Phys Med Rehabil. 2000;81(4):460-4.

25. Rejeski WJ, Martin K, Ettinger WH. Morgan T. Treating disability in knee osteoarthritis with exercise therapy: A central role for self-efficacy and pain. Arthritis Care Res. 1998;11(2):94-101.

26. Kurlowicz LH. Perceived Self-Efficacy, Functional Ability, and Depressive Symptoms in Older Elective Surgery Patients. Nurs Res. 1998;47(4):219-26. 
27. Waldrop D Jr, Lightsey OR, Ethington CA, Woemmel CA, Coke AL. Self-efficacy, optimism, health competence, and recovery from orthopedic surgery. J Couns Psychol. 2001;48(2):233-8.

28. Zhang L, Zhang M, Zhang J, Fu D. Self-efficacy on rehabilitative exercise and the influential factors in patients having undergone total hip or knee arthroplasty. Mod Clin Nurs. 2014;(1):5-8.

29. Li X, Lyu Q, Li J, Zhang M. Influence of exercise self-efficacy on exercise compliance after discharge of patients receiving hip arthroplasty. Chin Nurs Manage. 2019;19(09):1371-5.

30. Schulz KF, Altman DG, Moher D. [CONSORT 2010 Statement: updated guidelines for reporting parallel group randomised trials (Chinese version)]. Zhong Xi Yi Jie He Xue Bao. 2010;8(7):604-12.

31. Chan AW, Tetzlaff JM, Altman DG, Laupacis A, Gøtzsche PC, Krleža-Jerić K, et al. SPIRIT 2013 statement: defining standard protocol items for clinical trials. Rev Panam Salud Publica. 2015;38(6):506-14.

32. Bandura A. Social foundations of thought and action: A social cognitive theory.Social foundations of thought and action: A social cognitive theory. Englewood Cliffs: Prentice-Hall, Inc.; 1986.

33. Bandura A. Self-efficacy: The exercise of control. New York: W H Freeman/Times Books/ Henry Holt \& Co.; 1997.

34. Bandura A. Social Cognitive Theory in Cultural Context. Appl Psychol. 2002;51(2):269-90.

35. Bandura A. Health promotion by social cognitive means. Health Educ Behav. 2004;31(2):143-64.

36. Bandura A. Social learning theory. Oxford: Prentice-Hall; 1977.

37. Hørdam B, Sabroe S, Pedersen PU, Mejdahl S. Søballe K. Nursing intervention by telephone interviews of patients aged over 65 years after total hip replacement improves health status: a randomised clinical trial. Scand J Caring Sci. 2010;24(1):94-100.

38. Maisiak R, Austin J, Heck L. Health outcomes of two telephone interventions for patients with rheumatoid arthritis or osteoarthritis. Arthritis Rheum. 1996;39(8):1391-9.

39. Marcus AC, Garrett KM, Cella D, Wenzel LB, Brady MJ, Crane LA, et al. Telephone counseling of breast cancer patients after treatment: a description of a randomized clinical trial. Psychooncology. 1998;7(6):470-82.

40. Waldrop D Jr, Lightsey OR, Ethington CA, Woemmel CA, Coke AL. Self-efficacy, optimism, health competence, and recovery from orthopedic surgery. J Couns Psychol. 2001;48(2):233-8.

41. Downie WW, Leatham PA, Rhind VM, Wright V, Branco JA, Anderson JA. Studies with pain rating scales. Ann Rheum Dis. 1978;37(4):378-81.

42. Jensen MP, McFarland CA. Increasing the reliability and validity of pain intensity measurement in chronic pain patients. Pain. 1993;55(2):195-203.

43. Erden S, Arslan S, Deniz S, Kaya P. Gezer D. A review of postoperative pain assessment records of nurses. Appl Nurs Res. 2017;38:1-4.

44. Yen CF, Chiu T, Liou T, Liao H, Li Y, Liang C, et al. Does the planned long-term care policy in Taiwan meet the needs of people with disabilities? Health Policy. 2014;116(1):95-104. 
45. Federici S, Meloni F, Presti AL. International literature review on WHODAS II (World Health Organization Disability Assessment Schedule II). Life Span Disabil. 2009;12(1):83-110.

46. Meesters JJL, Verhoef J, Liem ISL, Putter H. Vliet Vlieland TPM. Validity and responsiveness of the World Health Organization Disability Assessment Schedule II to assess disability in rheumatoid arthritis patients. Rheumatology. 2010;49(2):326-33.

47. Chiu TY, Yen C, Chou C, Lin J, Hwang A, Liao H, Chi W. Development of traditional Chinese version of World Health Organization Disability Assessment Schedule 2.036 - item (WHODAS 2.0) in Taiwan: Validity and reliability analyses. Res Dev Disabil. 2014;35(11):2812-20.

48. WF Y. JM X. Application and evaluation of the Hospital Anxiety and Depression Scale in patients of general hospital. Chin J Behav Med Sci. 1993;(3):17-9.

49. Zigmond AS, Snaith RP. The Hospital Anxiety and Depression Scale. Acta Psychiatr Scand. 1983;67(6):361-70.

50. Watson R. A psychometric evaluation of the Chinese version of the Hospital Anxiety and Depression Scale in patients with coronary heart disease. J Clin Nurs. 2009;18(21):3068.

51. Söderman P, Malchau H. Is the Harris Hip Score System Useful to Study the Outcome of Total Hip Replacement? Clin Orthop Relat Res. 2001;384(3):189-97.

52. Davis KE, Ritter MA, Berend ME, Meding JB. The importance of range of motion after total hip arthroplasty. Clin Orthop Relat Res. 2007;465(465):180-4.

53. Harris WH. Traumatic Arthritis of the Hip After Dislocation and Acetabular Fractures: Treatment by Mold Arthroplasty. An End-Result Study Using a New Method of Result Evaluation. J Bone Joint Surg Am. 1969;51(4):737-55.

54. Ostendorf M, van Stel HF, Buskens E, Schrijvers AJP, Marting LN, Verbout AJ, et al. Patient-reported outcome in total hip replacement. A comparison of five instruments of health status. J Bone Joint Surg Br. 2004;86(6):801-8.

55. Salyers MP, Bosworth HB, Swanson JW, Lamb-Pagone J, Osher FC. Reliability and Validity of the SF12 Health Survey Among People With Severe Mental IIIness. Med Care. 2000;38(11):1141-50.

56. Shou J, Ren L, Wang H, Yan F, Cao X, Wang H, Wang Z, Zhu S, Liu Y. Reliability and validity of 12-item Short-Form health survey (SF-12) for the health status of Chinese community elderly population in Xujiahui district of Shanghai. Aging Clin Exp Res. 2016;28(2):339-46.

57. Dowsey MM, Castle DJ, Knowles SR, Monshat K, Salzberg MR, Choong PF. The effect of mindfulness training prior to total joint arthroplasty on post-operative pain and physical function: study protocol for a randomised controlled trial. Trials. 2014;15(1):208.

\section{Figures}




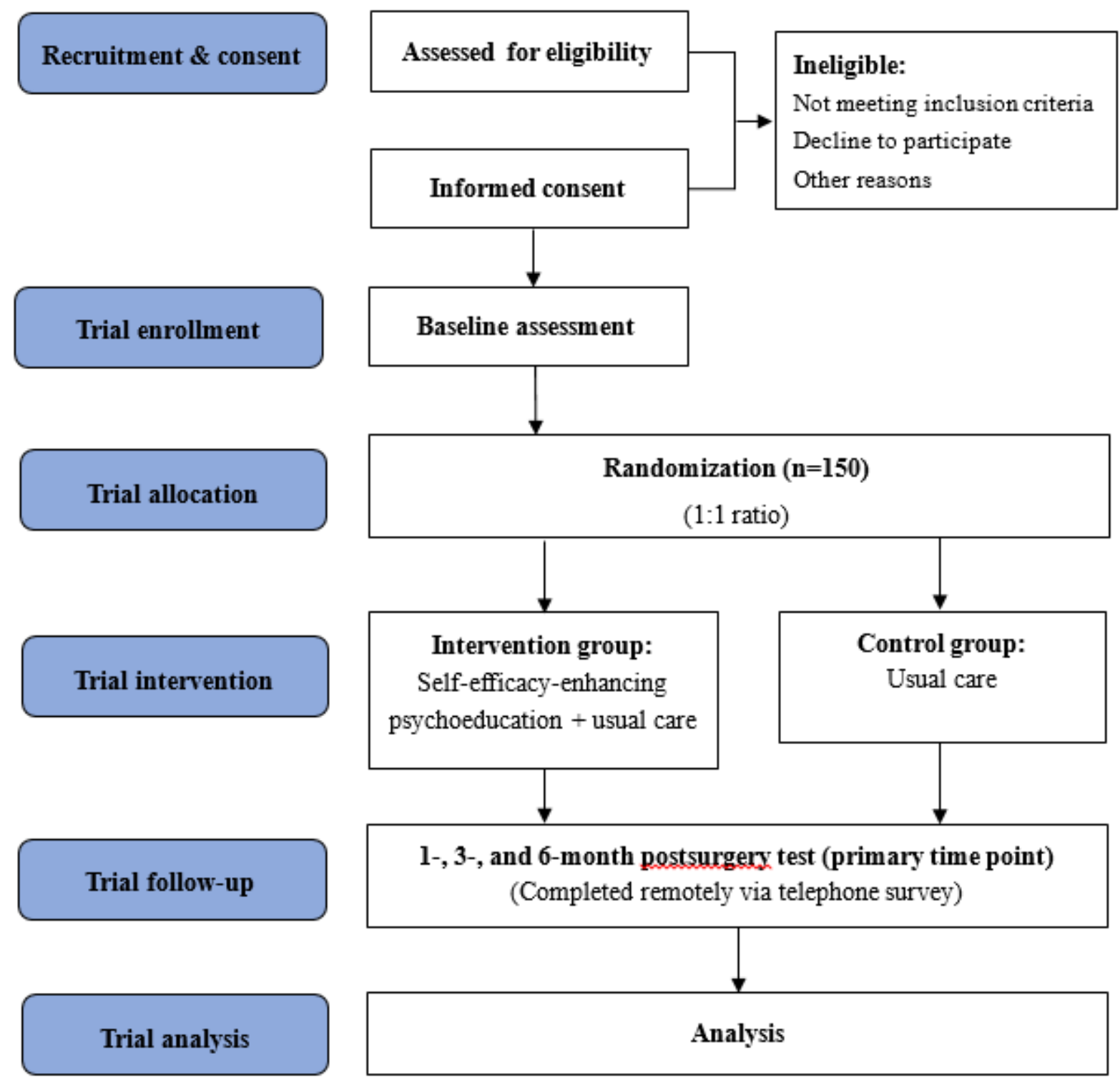

Figure 1

Flow diagram of the trial procedures. 